\title{
¿Sámaras o frutos samaroides? Estructura macroscópica de los frutos maduros de Myroxylon balsamum (Fabaceae, Papilionoideae)
}

\author{
¿Samaras or samaroid fruits? Macroscopic structure of mature \\ fruits of Myroxylon balsamum (Fabaceae, Papilionoideae)
}

Aguilar-Sandí, Diego ${ }^{1 *}$

1 Escuela de Biología, Universidad de Costa Rica, 11501 San Pedro, Montes de Oca, San José, Costa Rica.

*dd.10306@gmail.com

ORCID: https://orcid.org/0000-0003-3909-5425

\section{RESUMEN}

Las descripciones taxonómicas de Myroxylon balsamum mencionan que los frutos son samaroides o que son sámaras, pero no proporcionan una definición. En este artículo se describe la estructura de los frutos maduros de esta especie y se justifica la clasificación carpológica adoptada. También se incluyen observaciones sobre el contenido de la cámara seminífera y la calidad de las semillas. Se analizaron flores en distintos estados de desarrollo. Se asociaron las estructuras florales con las de los frutos. Estos son simples, secos, indehiscentes, glabros, alados y aplanados lateralmente. En posición proximal se ubica el pedicelo, el cáliz, el estípite y el ala; en posición distal la cámara seminífera, el estilo y el estigma. El ala es una prolongación de la pared externa del gineceo. En frutos sin semilla, la cámara seminífera es aplanada, el lóculo reducido y los depósitos de resina del mesocarpo conspicuos. En frutos con semilla, la cámara seminífera es subglobosa, el lóculo amplio y los depósitos de resina ocupan menor espacio. Las semillas pueden estar dañadas naturalmente. Los frutos de $M$. balsamum se clasifican como sámaras y no como frutos samaroides, ya que es posible diferenciar ambos tipos tomando en cuenta la estructura floral que origina el ala.

Palabras clave - Bálsamo; carpología; legumbre; morfología; Sophoreae.

Ref. bibliográfica: Aguilar-Sandí, D. 2020. “¿Sámaras o frutos samaroides? Estructura macroscópica de los frutos maduros de Myroxylon balsamum (Fabaceae, Papilionoideae)". Lilloa 57 (2): 89-98. Fundación Miguel Lillo, Tucumán, Argentina. D.O.I.: doi.org/10.30550/j.lil/2020.57.2/1

> Recibido: 15 agosto 2020 - Aceptado: 26 de noviembre 2020

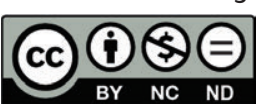

> URL de la revista: http://lilloa.lillo.org.ar

- Esta obra está bajo una Licencia Creative Commons Atribución - No Comercial - Sin Obra Derivada 4.0 Internacional. 


\begin{abstract}
The taxonomic descriptions of Myroxylon balsamum mention that the fruits are samaroids or samaras, but without providing a definition. In this article, the macroscopic structure of the mature fruits of this species is described and the carpological classification adopted is justified. Observations on the content of the seed-chamber and the quality of the seeds are also included. Flowers in different stages of development were analyzed. The floral structures were associated with the structures of the fruits. The fruits are simple, dry, indehiscent, glabrous, winged and laterally flattened. The pedicel, calyx, stipe and wing are in a proximal position, while the seed-chamber, style and stigma are distally located. The wing is an extension of the external wall of the gynoecium. In seedless fruits, the seed-chamber is flattened, the locule is reduced and the mesocarp resin deposits are conspicuous. In fruits with seeds, the seed-chamber is subglobose, the loculum is wide and the resin deposits occupy less space. The seeds may be damaged naturally. The fruits of M. balsamum are classified as samaras and not as samaroid fruits, since it is possible to differentiate both types by taking into account the floral structure that originates the wing.
\end{abstract}

Keywords - Balsam; carpology; legume; morphology; Sophoreae.

\title{
INTRODUCCIÓN
}

Los frutos de las leguminosas presentan alta diversificación morfológica que incluye vainas, folículos, núculas, sámaras, drupas, lomentos y craspedios (Córdula, Morim, Alves, 2014; The Legume Phylogeny Working Group [LPWG], 2017). Sin embargo, nunca ha existido consenso sobre la clasificación de los frutos de las angiospermas (Font Quer, 2007; Judd, Campbell, Kellogg, Stevens, Donoghue, 2016). En el caso de las sámaras, estas se han definido y clasificado con base en el número de semillas, la estructura floral que origina el ala y su posición respecto a la cámara seminífera u ovario desarrollado. Algunos autores diferencian entre sámaras, frutos samaroides y samarios, pero sin lograr acuerdo en la definición de cada tipo; aunque coinciden en que el ala representa una adaptación para la dispersión por viento (Vidal, 1978; Mirle y Burnham, 1999; Manchester y O'Leary, 2010).

Myroxylon L. f. (Fabaceae, Papilionoideae) es un género de árboles que comprende dos especies, $M$. balsamum (L.) Harms y M. peruiferum L. f., cuya distribución geográfica abarca el sur de México, Centroamérica y el norte de Suramérica (Sartori, Lewis, Mansano, Tozzi, 2015). Tradicionalmente se ha clasificado en la tribu Sophoreae, grupo polifilético que agrupa géneros cuyas flores presentan distintos grados de simetría bilateral, pétalos poco diferenciados y estambres libres, caracteres que han sido interpretados como basales o como reversiones evolutivas (Tucker, 1993; Sartori y Tozzi, 2002; Cardoso et al., 2012).

En Costa Rica, $M$. balsamum se encuentran de forma natural en zonas bajas de la vertiente pacífica (0-850 $\mathrm{m}$ snm), aunque es posible encontrar árboles cultivados en el Valle Central, sobre los $900 \mathrm{~m} \mathrm{snm}$. Su nombre vernáculo es bálsamo; vegetativa- 
mente se reconoce por la presencia de peridermis grisácea, profusamente lenticelada, corteza resinosa, estípulas deciduas o ausentes, hojas alternas imparipinnadas, folíolos alternos, con líneas y puntos translúcidos, y ápices acuminados (Aguilar-Sandí, 2020). De los árboles de esta especie se obtiene el bálsamo del Perú, resina con múltiples usos que ha sido comercializada desde la época precolombina, siendo El Salvador el principal país productor (Martínez-Rivera, Blanco, Colorado, Guerra, AntonioSerrato, 2015). En Costa Rica, los bálsamos se consideran en peligro de extinción debido a que sus poblaciones naturales son reducidas y han experimentado presión por tala (Jiménez, 1998). No obstante, aún no han sido incluidos en la Lista Roja de la Unión Internacional para la Conservación de la Naturaleza (IUCN, 2020).

Se ha estudiado la organogénesis de las flores (Tucker, 1993) y la anatomía de los folíolos de esta especie (Sartori y Tozzi, 2002). Sin embargo, no se encontraron trabajos sobre la estructura de sus frutos. Vidal (1978) describió la morfología externa del fruto de Myroxylon, pero lo hizo a partir de material botánico de M. peruiferum. En las descripciones taxonómicas de M. balsamum se menciona que el fruto es samaroide (Rudd, 1968; Zamora, 2010) o que es una sámara (Sartori et al., 2015), pero en ningún caso se definen estos términos; tampoco se discuten los alcances de la clasificación asumida.

Considerando lo anterior, en este artículo se describe la estructura macroscópica de los frutos maduros de $M$. balsamum y se da una justificación de la clasificación carpológica adoptada. Adicionalmente, se incluyen observaciones sobre el contenido de la cámara seminífera y la calidad de las semillas.

\section{MATERIALES Y MÉTODOS}

Durante noviembre y diciembre de 2019 se recolectaron flores y frutos de tres árboles de M. balsamum ubicados en sitios urbanos de la provincia de San José, Costa Rica. El primer árbol [altura $(\mathrm{h})=28 \mathrm{~m}$, diámetro a la altura del pecho $(\mathrm{DAP})=$ $85 \mathrm{~cm}$ )], se encuentra en el parqueo de un centro comercial en el cantón de Santa Ana (0955'58,6”N 84¹0'42,6”O). Los otros en el cantón de Montes de Oca, uno en el Jardín Botánico José María Orozco $(\mathrm{h}=38 \mathrm{~m}, \mathrm{DAP}=106 \mathrm{~cm}$; 0956’07,3”N $84^{\circ} 03^{\prime} 05,7$ 'O) y el otro en la Ciudad de la Investigación $(\mathrm{h}=16 \mathrm{~m}, \mathrm{DAP}=95 \mathrm{~cm}$; $\left.9^{\circ} 56^{\prime} 24,5^{\prime \prime} \mathrm{N} 84^{\circ} 02^{\prime} 40,8^{\prime} \mathrm{O}\right)$. Estos dos sitios forman parte del campus de la Universidad de Costa Rica.

Se analizaron flores en diferentes estados de desarrollo. Las estructuras florales se asociaron con las estructuras de los frutos maduros; para describirlas se tomó en cuenta la terminología utilizada por Rudd (1968), Vidal (1978) y Sartori et al. (2015). Se diseccionó la cámara seminífera de 800 frutos seleccionados aleatoriamente. Se describió su estructura interna. Adicionalmente, se contabilizó el número de semillas y se registró su calidad (en buen estado o dañadas). Los porcentajes obtenidos se compararon mediante pruebas chi cuadrado realizadas con el programa estadístico R (R Core Team, 2020). Las fotografías se tomaron con una cámara digital Nikon D3200. 


\section{Material testigo}

COSTA RICA. Prov. San José, Cantón Santa Ana, Distrito Santa Ana, parqueo de la plaza Koros, $09^{\circ} 55^{\prime} 58,6^{\prime \prime} \mathrm{N} 84^{\circ} 10^{\prime} 42,66^{\prime} \mathrm{O}, 900 \mathrm{~m}$ snm, 21-IV-2019 (fr), Aguilar-Sandí 13 (USJ).

COSTA RICA. Prov. San José, Cantón Montes de Oca, Distrito San Pedro, Jardín Botánico José María Orozco, Universidad de Costa Rica [0956’07,3”N 8403’05,7”O], $1200 \mathrm{~m}$ snm, 26-IX-2012 (fr), Morales 2345 (USJ).

COSTA RICA. Prov. San José, Cantón Montes de Oca, Distrito San Pedro, Ciudad de la Investigación, Universidad de Costa Rica, 956'24,5"N 8402'40,8”O, 1295 m snm, 12-XII-2019 (fl, fr), Aguilar-Sandí 17 (USJ).

\section{RESULTADOS}

Las flores de M. balsamum son pediceladas, zigomorfas y bisexuales; presentan cinco sépalos fusionados, cinco pétalos libres, uno adaxial amplio (estandarte) y cuatro lineares poco diferenciados (dos laterales y dos abaxiales), diez estambres con anteras dorsifijas, gineceo estipitado, ovario súpero, glabro y unicarpelar. Conforme el gineceo se desarrolla, el resto de las partes florales se marchitan (Fig. 1A).

Los frutos maduros son simples, secos, indehiscentes, glabros, alados y aplanados lateralmente. Conservan remanentes de todas las estructuras florales, a excepción de la corola y el androceo. En posición proximal se encuentra el pedicelo, el cáliz, el estípite y el ala. En posición distal, la cámara seminífera, el estilo y el estigma (Fig. 1B). El ala es una prolongación de la pared externa del gineceo, ubicada entre el estípite y el ovario, tiene textura papirácea y presenta dos secciones asimétricas separadas por una sección de tejido vascular. La cámara seminífera corresponde al ovario desarrollado y tiene textura coriácea. Cortándola transversalmente se observa el exocarpo, el mesocarpo con depósitos de resina, el endocarpo y el lóculo (Fig. $1 \mathrm{C})$.

Al madurar, los frutos tienen tonalidades amarillentas y brillosas, que cambian a grisáceo deslustradas según el tiempo que lleven en el suelo; presentan tamaños de $4(7)-9(12) \times 1,2(2)-3,2(4) \mathrm{cm}$, incluyendo el ala, que puede ser recta o curva. Cuando son dispersados pierden el pedicelo, el cáliz y en algunos casos el estípite (Fig. 1D). El 92,5 \% de los frutos con semilla presentó una, mientras que el restante $7,5 \%$ presentó dos (Fig. 1E); valores que son contrastantes entre sí $\left(\chi^{2}=72,25\right.$; g.l = $1 ; \mathrm{P}<0,001)$.

En frutos sin semilla, la cámara seminífera es aplanada, el lóculo reducido y los depósitos de resina del mesocarpo conspicuos (Fig. 2A). En frutos con semilla, la cámara seminífera es subglobosa, el lóculo amplio y los depósitos de resina ocupan menor espacio (Fig. 2B). Se encontraron diferencias en los porcentajes de frutos sin semilla (16 \%), con semilla en buen estado (59\%) y con semilla dañada $(25 \%)\left(\chi^{2}=\right.$ $30,10 ;$ g.l. $=2 ; \mathrm{P}<0,001)$. Las semillas en buen estado son reniformes, lisas, suaves y blanquecinas; externamente es posible identificar el extremo donde se ubica el embrión, la zona del hilo y los cotiledones (Fig. 3A), mientras que las semillas da- 

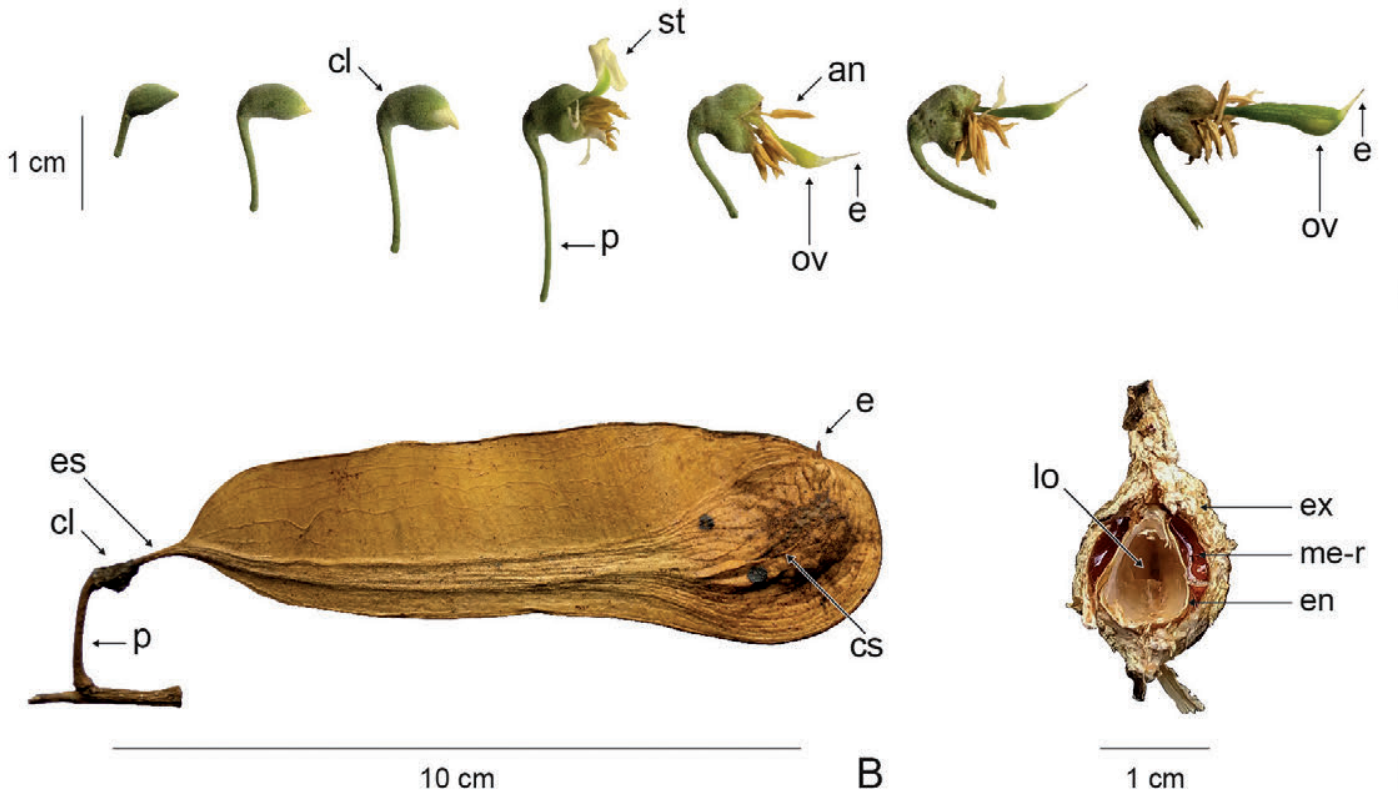

$B$

C
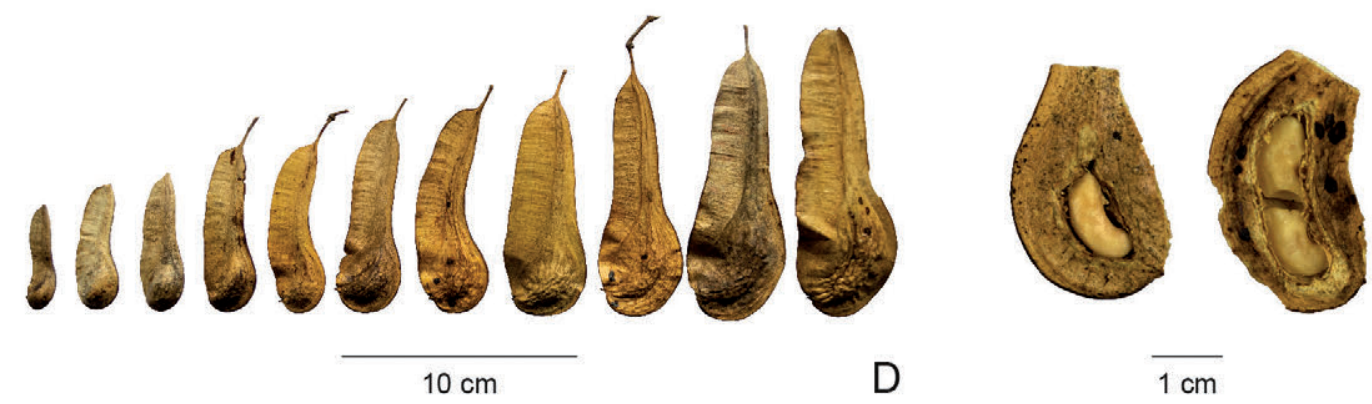

Fig. 1. Myroxylon balsamum. A) Desarrollo de las flores: botón floral, antesis, marchitamiento, crecimiento del gineceo. B) Vista lateral del fruto. C) Corte transversal de la cámara seminífera. D) Variación en el tamaño de los frutos. E) Corte longitudinal de la cámara seminífera (se muestran las semillas). Abreviaturas: an: antera, cl: cáliz, cs: cámara seminífera, e: estilo, en: endocarpo, es: estípite, ex: exocarpo, lo: lóculo, me-r: mesocarpo-resina, p: pedicelo, st: estandarte, ov: ovario. Aguilar-Sandí 13 (USJ).

Fig. 1. Myroxylon balsamum. A) Development of the flowers: flower bud, anthesis, wilting, growth of the gynoecium. B) Side view of the fruit. C) Cross-section of the seed-chamber. D) Variation in fruit size. E) Longitudinal section of the seed-chamber (the seeds are shown). Abbreviations: an: anther, cl: calyx, cs: seed-chamber, e: style, en: endocarp, es: stipe, ex: exocarp, lo: locule, me-r: mesocarp-resin, p: pedicel, st: standard petal, ov: ovary. Aguilar-Sandí 13 (USJ).

ñadas muestran tonalidades amarillentas, parduzcas y negras, desintegración de los cotiledones y crecimiento anómalo (Fig. 3B).

\section{DISCUSIÓN}

Vidal (1978) definió la sámara como el fruto seco indehiscente que presenta de una a cuatro semillas y un ala formada a partir de la pared del ovario. Este autor diferenció entre sámaras simples, samarios y frutos samaroides. El primer tipo se origina de un ovario libre, unicarpelar, y desarrolla un ala circular o lateral, que en este último 

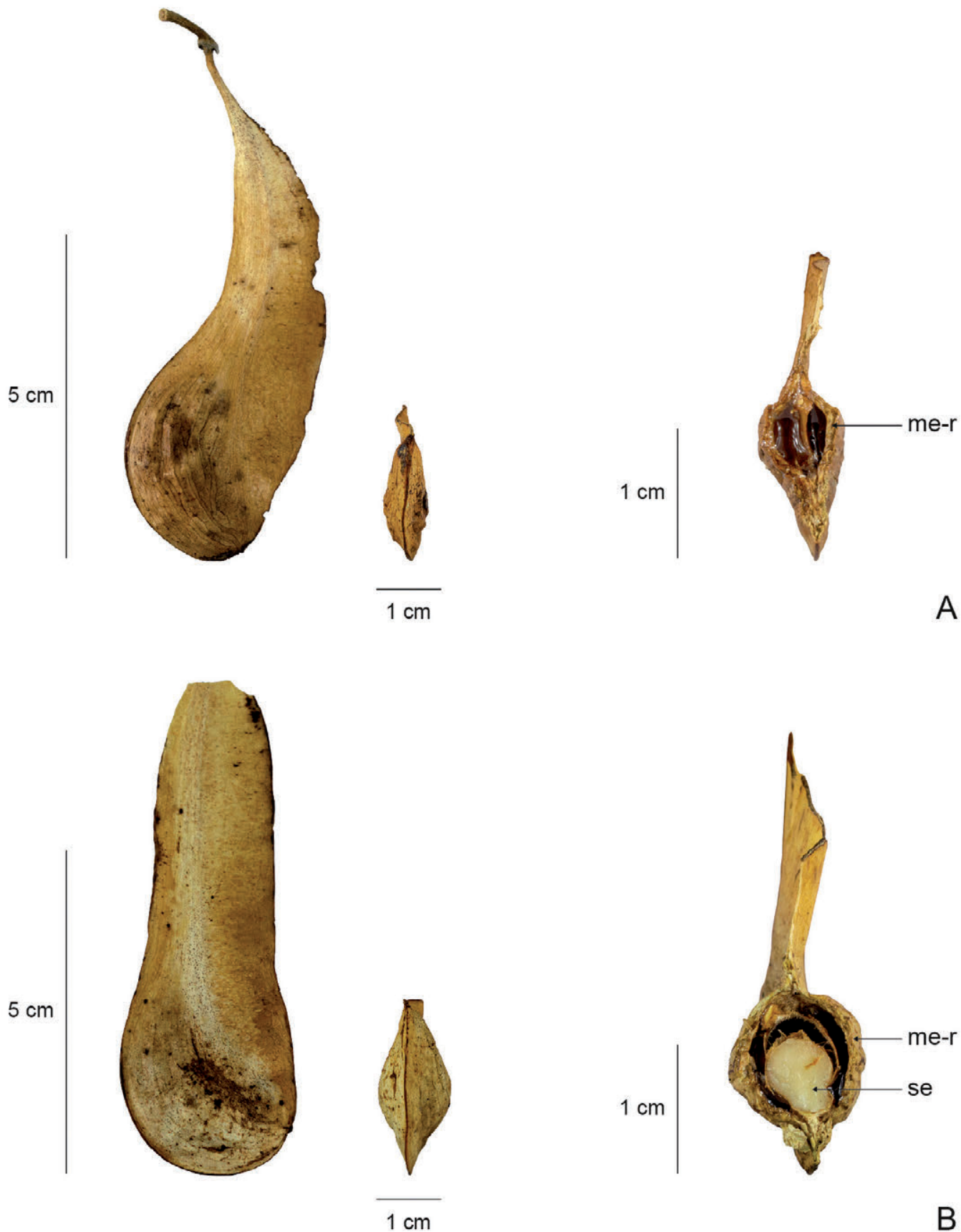

Fig. 2. Myroxylon balsamum. A) Fruto sin semilla. B) Fruto con semilla en buen estado. Abreviaturas: me-r: mesocarpo-resina, se: semilla. Aguilar-Sandí 13 (USJ).

Fig. 2. Myroxylon balsamum. A) Seedless fruit. B) Fruit with seed in good condition. Abbreviations: me-r: mesocarp-resin, se: seed. Aguilar-Sandí 13 (USJ).

caso puede ser apical (distal) o basal (proximal). El samario corresponde al mericarpo de un esquizocarpo, mientras que el fruto samaroide es aquel cuya ala se forma de una estructura floral distinta del ovario. Vidal (1978) estudió las sámaras simples de ala lateral y las reconoció en 19 géneros, incluido Myroxylon, de siete familias (Anacardiaceae, Casuarinaceae, Celastraceae, Fabaceae, Phytolaccaceae, Polygalaceae y Ulmaceae). 

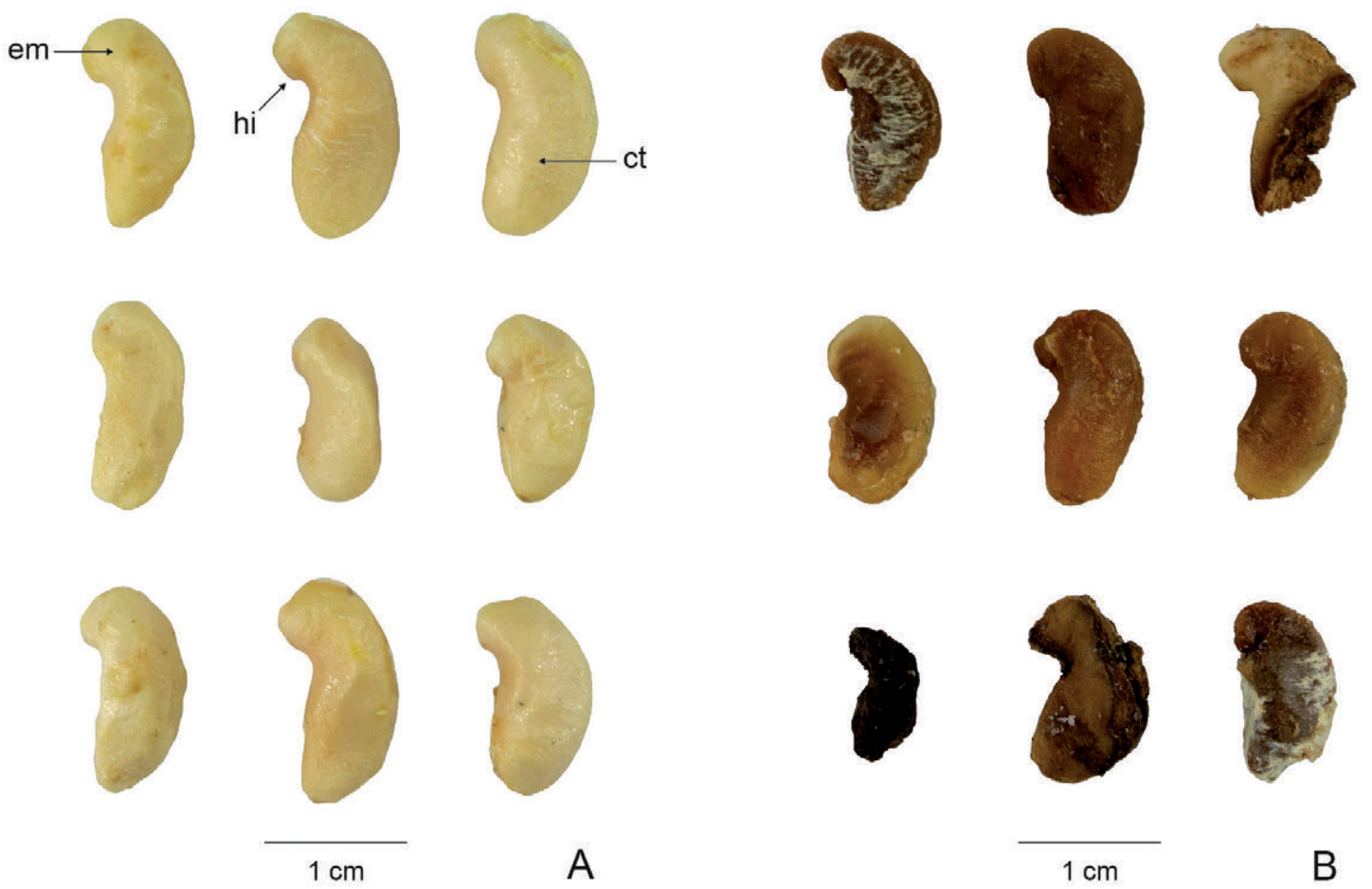

A

$1 \mathrm{~cm}$

B

Fig. 3. Myroxylon balsamum. A) Semillas en buen estado. B) Semillas dañadas. Abreviaturas: em: localización del embrión, hi: hilo, ct: cotiledón. Aguilar-Sandí 13 (USJ).

Fig. 3. Myroxylon balsamum. A) Seeds in good condition. B) Damaged seeds. Abbreviations: em: location of the embryo, hi: hilum, ct: cotyledon. Aguilar-Sandí 13 (USJ).

Mirle y Burnham (1999) estudiaron las sámaras asimétricas; definiéndolas como el fruto, o la parte de un fruto, con el ala extendida hacia un lado del ovario, identificándolas en 39 géneros, incluido Myroxylon, de 11 familias (Aceraceae, Anacardiaceae, Fabaceae, Malpighiaceae, Phytolaccaceae, Polygalaceae, Polygonaceae, Rutaceae, Sapindaceae, Trigoniaceae y Ulmaceae). Font Quer (2007) consideró que la sámara es todo aquenio con una o más alas. Mientras que para Manchester y O'Leary (2010), la sámara es un fruto indehiscente monospermo con el ala formada a partir del ovario; en tanto que el fruto samaroide presenta más de una semilla.

Las características morfológicas de los frutos de M. balsamum coinciden con la definición de sámara simple sensu Vidal (1978). Razón por la cual se acepta aquí dicha clasificación; misma que es compatible con el hecho de que estos frutos son vainas que desarrollan un ala proximal conforme maduran. Si bien la vaina, o legumbre, se ha definido como un fruto seco dehiscente originado de un ovario unicarpelar que se abre por dos suturas (Font Quer, 2007; Flores-Vindas, 2013), también se acepta que las vainas pueden ser indehiscentes y que, en general, el pericarpo de los frutos de las leguminosas puede presentar modificaciones (Córdula et al., 2014). No se recomienda clasificar los frutos de los árboles de bálsamo como samaroides, criterio que siguieron Rudd (1968) y Zamora (2010), pero sí como sámaras, tal cual hicieron Sartori et al. (2015). Sin embargo, estos autores no aportaron una definición que permitiera saber qué entendieron por sámara. 
La presencia de piezas florales remanentes en los frutos maduros es común en muchas especies, ya sea porque forman parte integral del fruto o porque son estructuras accesorias (Scagel et al., 1977; Weberling, 1989); en cuyo caso pueden tener importancia taxonómica. Por ejemplo, Mirle y Burnham (1999) establecieron que el estilo vestigial es una característica útil para identificar el género al que pertenecen las sámaras que han sido dispersadas, incluidas las de Myroxylon. Por otra parte, en las descripciones morfológicas de $M$. balsamum no se hace referencia a la existencia de depósitos de resina en el mesocarpo, dándose únicamente información sobre la resina de la corteza (Rudd, 1968; Zamora, 2010; Sartori et al., 2015). No obstante, la resina del mesocarpo no ha pasado desapercibida en medicina tradicional, pues se recomienda realizar preparaciones alcohólicas a base de frutos fragmentados para tratar cefaleas y dolores reumáticos mediante frotaciones (Ñ́nez, 1978).

Rudd (1968) consideró que el tamaño de los frutos permitía diferenciar las variedades de esta especie, particularmente, $M$. balsamum var. balsamum (con frutos de $8-11 \mathrm{~cm}$ de largo) y $M$. balsamum var. pereirae (con frutos de 6-8 cm de largo). Sin embargo, Sartori et al. (2015) no reconocieron estas entidades taxonómicas por no existir caracteres consistentes para delimitarlas; razonamiento que se confirma aquí, ya que la longitud de los frutos producidos por un mismo árbol oscila entre 4 y 12 $\mathrm{cm}$. Por otra parte, Sartori et al. (2015) consideraron que el fruto de M. balsamum era monospermo, mientras que Rudd (1968) notó la existencia de frutos con dos semillas y lo poco frecuente de su ocurrencia.

La formación de frutos sin semilla o con semillas dañadas es usual en plantas, siendo la partenocarpia, el aborto de semillas y la depredación pre-dispersión las causas que permite dar una explicación de ello (López-Almansa y Gil, 2003; Östergard, Hambäck, Ehrlén, 2007). El aborto puede deberse a deficiencias en la polinización, a limitaciones en los recursos disponibles o a variaciones en el éxito reproductivo de los organismos progenitores (Bawa y Webb, 1984). No obstante, no se encontraron trabajos sobre la variación en el contenido de la cámara seminífera y la calidad de las semillas de $M$. balsamum, lo que indica que este es un campo aún inexplorado.

Si bien, el grado de urbanización, la fragmentación del hábitat y la reducción poblacional influyen en la producción de semillas (Nagamitsu, Kikuchi, Hotta, Kenta, Hiura, 2014), no puede asumirse que estos factores expliquen que aproximadamente el $40 \%$ de los frutos carecieran de semillas o las tuvieran dañadas, a pesar de que los árboles progenitores crezcan en sitios urbanos.

\section{CONCLUSIÓN}

Existe suficiente evidencia morfológica y argumentos teóricos para clasificar los frutos de los árboles de $M$. balsamum como sámaras y no como frutos samaroides, ya que es posible diferenciar ambos tipos tomando en cuenta la estructura floral que origina el ala. Si bien pueden surgir diferencias de criterio sobre la clasificación carpológica a seguir, se recomienda respaldar la clasificación elegida con una definición que no dé lugar a ambigüedades, ya sea mencionándola explícitamente o haciendo referencia a una fuente bibliográfica previa. 
Las observaciones realizadas sobre la variación en el contenido de la cámara seminífera y calidad de las semillas pueden ser tomadas en cuenta en programas de silvicultura. Sin embargo, para dar una explicación satisfactoria de esa variación se hace necesario poner a prueba hipótesis ecológicas y evolutivas; tema que debe estudiarse con mayor profundidad en futuras investigaciones.

\section{AGRADECIMIENTOS}

Este artículo se deriva de los estudios que realicé en el programa de Licenciatura en Biología con énfasis en Botánica de la Escuela de Biología de la Universidad de Costa Rica. Le expreso mi agradecimiento a Elmer G. García por haber revisado la primera versión del manuscrito, sus sugerencias influyeron de forma decisiva en el texto final.

\section{BIBLIOGRAFÍA}

Aguilar-Sandí, D. (2020). Sinopsis del género Myroxylon (Fabaceae) en Costa Rica. Phytoneuron 2020-20: 1-10.

Bawa, K. S. y Webb, C. J. (1984). Flower, fruit and seed abortion in tropical forest trees: implications for the evolution of paternal and maternal reproductive patterns. American Fournal of Botany 71 (5): 736-751.

Cardoso, D., De Queiro, L. P., Pennington, R. T., De lima, H. C., Fonty, E., Wojciechowski, M. F. y Lavin, M. (2012). Revisiting the phylogeny of papilionoid legumes: new insights from comprehensively sampled early-branching lineages. American Fournal of Botany 99 (12): 1991-2013. doi: 10.3732/ajb.1200380

Córdula, E., Morim, M. P. y Alves, M. (2014). Morfologia de frutos e sementes de Fabaceae ocorrentes em uma área prioritária para a conservação da Caatinga em Pernambuco, Brasil. Rodriguésia 65 (2): 505-516.

Flores-Vindas, E. (2013). La planta, estructura y función (4ta ed.). Cartago, Costa Rica: Editorial Tecnológica de Costa Rica.

Font Quer, P. (2007). Diccionario de botánica (3ra ed.). Barcelona, España: Península.

Jiménez, Q. (1998). Árboles maderables en peligro de extinción en Costa Rica (2da ed.). Heredia, Costa Rica: Instituto Nacional de Biodiversidad.

Judd, W. S., Campbell, C. S., Kellogg, E. A., Stevens, P. F. y Donoghue, M. J. (2016). Plant Systematics: A Phylogenetic Approach (4th ed). Massachusetts, USA: Sinauer.

IUCN. (2020). The IUCN Red List of Threatened Species. Version 2020-2. Recuperado de https://www.iucnredlist.org

López-Almansa, J. C. y Gil, L. (2003). Empty samara and parthenocarpy in Ulmus minor s.1. in Spain. Silvae Genetica 52 (5-6): 241-243.

LPWG. (2017). A new subfamily classification of the Leguminosae based on a taxonomically comprehensive phylogeny. Taxon 66: 44-77. doi: 10.12705/661.3 
Manchester, S. R. y O'Leary, E. L. (2010). Phylogenetic distribution and identification of fin-winged fruits. Botanical Review 76: 1-82. doi: 10.1007/s 12229-0 $10-9041-0$

Martínez-Rivera, J. C., Blanco, J. A., Colorado, R. A., Guerra, O. E. y Antonio-Serrato, H. D. (2015). El impacto cultural del árbol de bálsamo en Chiltiupán, La Libertad, El Salvador. Entorno 58: 25-33.

Mirle, C. y Burnham, R. J. (1999). Identification of asymmetrically winged samaras from the Western Hemisphere. Brittonia 51 (1): 1-14.

Nagamitsu, T., Kikuchi, S., Hotta, M., Kenta, T. y Hiura, T. (2014). Effects of population size, forest fragmentation, and urbanization on seed production and gene flow in an endangered Maple (Acer miyabei). The American Midland Naturalist 172 (2): 303-316.

Núnez, E. (1978). Plantas medicinales de Costa Rica y su folclore (2da ed.). San José, Costa Rica: Editorial de la Universidad de Costa Rica.

Östergard, H., Hambäck, P. A. y Ehrlén, J. (2007). Pre-dispersal seed predation: the role of fruit abortion and selective oviposition. Ecology 88 (12): 2959-2965.

R Core Team. (2020). R: A language and environment for statistical computing. Vienna, Austria: R Foundation for Statistical Compunting. https://www.R-project.org/.

Rudd, V. E. (1968). Leguminosae of Mexico-Faboideae. I. Sophoreae and Podalyrieae. Rhodora 70: 492-532.

Sartori, A. L. B. y Tozzi, A. M. G. A. (2002). Comparative leaflet anatomy in Myrocarpus Allemão, Myroxylon L. f and Myrospermum Jacq. (Leguminosae-Papilionoideae-Sophoreae) species. Botanical Fournal of the Linnean Society 140: 249-259.

Sartori, A. L. B., Lewis, G. P., Mansano, V. F. y Tozzi, A. M. G. A. (2015). A revision of the genus Myroxylon (Leguminoseae: Papilionoideae). Kew Bulletin 70: 48. doi: 10.1007/S12225-015-9604-7

Scagel, R. F., Bandoni, R. J., Rouse, G. E., Schophield, W. B., Stein, J. R. y Taylor, T. M. C. (1977). El reino vegetal, los grupos de plantas y sus relaciones evolutivas. Barcelona, España: Ediciones Omega.

Tucker, S. C. (1993). Floral ontogeny in Sophoreae (Leguminosae: Papilionoideae). I. Myroxylon (Myroxylon group) and Castanospermum (Angylocalyx group). American Fournal of Botany 80 (1): 65-75.

Vidal, W. N. (1978). Considerações sobre as sâmaras que têm ala paranuclear. Rodriguésia 30 (47): 109-168.

Weberling, F. (1989). Morphology of flowers and inflorescences. Cambridge, United Kingdom: Cambridge University Press.

Zamora, N. (2010). Fabaceae. En: B. E. Hammel, M. H. Grayum, C. Herrera, N. Zamora (Eds.). Manual de Plantas de Costa Rica. Vol. 5. (pp. 395-775). Monographs in Systematic Botany from the Missouri Botanical Garden, 119. 